\title{
On How Designers Communicate the Functionality of Articulated Product Concepts in Sketches
}

\author{
G Kalyan Ramana ${ }^{1}$, Prasad S Onkar ${ }^{2}$ \\ ${ }^{1}$ Department of Design, Indian Institute of Technology, Hyderabad \\ gkalyanramana@gmail.com \\ ${ }^{2}$ Department of Design, Indian Institute of Technology, Hyderabad \\ psonkar@iith.ac.in
}

\begin{abstract}
This work describes the investigations on how designers communicate functionality of articulated product concepts through sketching. Sketching has been used by designers to explore concepts in the early phases of design. In articulated product concept sketches, the ideas represented through sketching goes through several iterations before it is realized into physical products. This iterative process increases the overall product development time. One of the primary reasons for a longer product development cycle is that there is a great difficulty in visualizing motions of articulated product concepts through sketching. Designers use annotations to represent motion details which need to be validated for physically realizable ideas. To study this, design experiments were conducted which involved four designers to sketch concepts for an articulated product. Think-aloud protocol analysis methodology was followed. The sketching activity is video recorded and transcribed with a modified coding scheme for sketch analysis. To quantify the amount of effort in representing motion, a measure of effort metric is introduced which is derived from the coding scheme. The analysis shows that there is considerable amount of effort from the designers in representing the motion in their sketches. It is also observed that conventional paper-based sketching does not support complex motion explorations. These observations form the basis for development of novel sketch-based interfaces to support a variety of explorations of articulated product concepts.
\end{abstract}

\section{Keywords: Functionality of Articulation, Product sketch, Fraction of effort}

\section{Introduction}

Sketching is used typically by a variety of professionals like architects, artists, engineers etc. to design/plan their work so that they can externalize their ideas into concepts. Such design sketches can be broadly classified into two categories viz., talking sketches and thinking sketches (Ferguson, 1994). Talking sketches are drawings and visualizations that showcase their concepts and thinking sketches are predominantly used for creative explorations and ideation. Similar observations are also made in the literature (Ferguson, 1994) (Gero \& Purcell, 1998) which also emphasizes advantages of sketching in the design process. One of the advantages is that sketching facilitates to reduce load on short-term memory by offloading part of imagery onto physical media (Bilda, Gero, \& Purcell, 2006). This process of transfer helps 


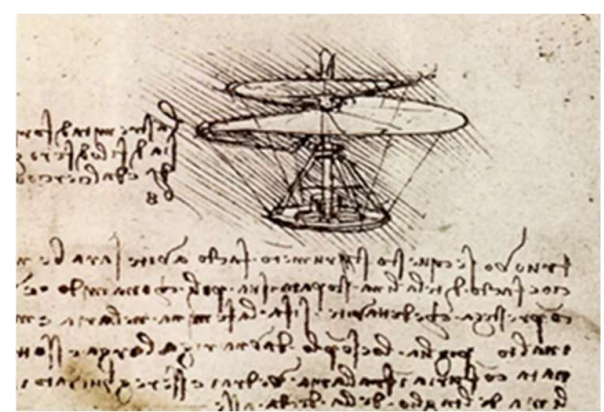

(a)

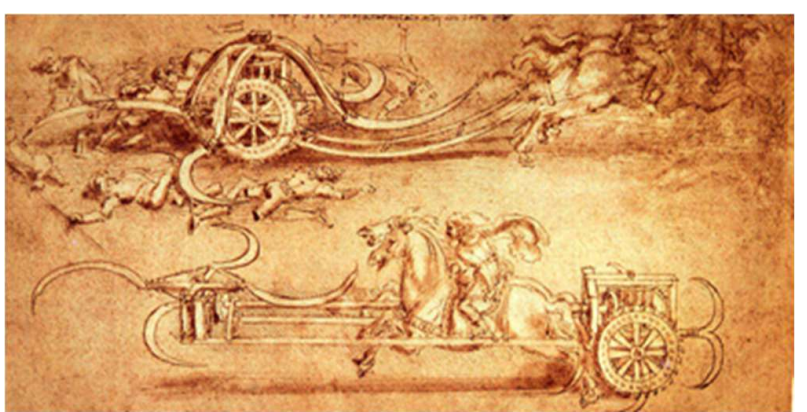

(b)

Figure 1: (a) Airscrew (b) Scythed Chariot

the designer to record form/shape, spatial organisation, spatio-temporal configuration of components being visualised. Thus, being an efficient way to record ideas quickly. But when sketches are used for analysing the conceptual validity, especially when the concepts have moving components, it would be difficult to comprehend multiple motions of several components instantaneously. Hence, it can be argued that if the designers are provided with an interactive digital sketching environment in which they can ascertain the motion of connected sketched components then, it will enhance their creative exploration.

To support this argument, first it must be identified as how the existing paper-based sketching facilitates development of concept which involves relative motion of its components. It is observed that designers represent motion through different annotations in their sketch and most of the concepts involve simple motions like rotation about a point and translation along a line. Hence, it is important to understand the time and amount of effort involved in both communicating the motion of articulated product concepts and exploring novel motions of the parts. The argument here is that if we understand the process of annotation, which in turn, represent motions, then we will be able to support concept exploration involving relative motion better. So, to understand the phenomena, design experiments were conducted to estimate the effort involved in representing motions (primarily through annotations) and complexity of the motion of the sketched components. This work focuses on the analysis of the sketching activity of product designs for communicating the functionality of articulations in the concept. The word 'articulations' here refer to the joints that are present between objects. These articulations facilitate relative motion between objects. For example, the propeller in Fig. 1(a) is held vertically on a base and rotates about a vertical axis.

The main problem in communicating functionality of articulations in the concept in a product sketch is difficulty for the designer or the observer (clients, designers or engineers) to comprehend or coherently follow the explanation. This makes the designer's task difficult and time consuming thereby considerable effort is made for explanation. For example, consider the air screw sketch by Leonardo da Vinci shown in Fig. 1(a). If a person has to explain the working principle of the air screw by looking at the sketch, it is difficult to comprehend because of the complex relative motion between sketched components. The explanation is also difficult because it has multiple connected parts. Moreover, difficulty level depends on the way the sketched components are connected (also called kinematic joints (Norton, 2004)). As said earlier, simple motions like rotations and translations can be easily conveyed but their combination (complex motion) is not so easy. Also, if the concept sketch is given to another designer then, the latter may not be able to understand its functionality completely unless it is simulated. For example, in Fig. 1(a), designers (Leonardo in this case) use annotations or words for motion representation which do not reveal complete information. 
Essentially, an articulated product sketch is an embodied depiction of a mechanism design problem (Norton, 2004). For example, consider the scythed chariot shown in Fig. 1(b). The swords in front are horses are rotated (output motion) by a mechanism whose input motion is driven from rotating wheels. The motion from the wheels to the swords are assumed to be transmitted by gears (in Fig. 1(b), these gears are shown partially). The intended motion of the sketched components can only be verified/realized by simulating computer models or by making a physical prototype. So, the motion patterns assumed by the designers and represented in their sketches may or may not be realizable. This results in ambiguity which restricts further explorations.

\section{Literature Review}

Conceptual design is an important phase in the overall design process because of its influence on the performance parameters of the final outcome (Boothroyd, Dewhurst, \& Knight, 2010) . Typically, designers use sketching as a medium to explore concepts in the early stages of design because of its inherent advantages (Fish \& Scrivener, 1990) and (Gero \& Purcell, 1998). Literature suggests that there has been an effort to understand the conceptual design process by analyzing the sketches created by the designers. Several theories have been proposed in literature to explain the importance of the sketching in the design process. It is argued that, in the early phases of conceptual design, sketching is like reflective conversations with medium and the self (Schon, 2017) and as a dialogue with the designer himself/herself (Goldschmidt, 1991). (Gero \& Kannengiesser, 2004) gave ontology for the concept generation framework of design actions. In this work, one can see how structure of product sketch evolves through the designer's expectations and it functions. At every stage in the design process, the designer always tries to bridge the gap between expected behavior and actual behavior by making changes in the product structure thus, reflecting a change in function and behavior.

A statistical analysis is explored in (Onkar \& Sen, 2010) to elucidate the underlying product structure. There are many ways in which sketching supports the conceptual design activity, but it is important to understand limitations of traditional sketching (in the context of conceptual actions) and further develop technological support to augment the computational sketching system. Such systems have been developed and enlisted in (Jorge \& Samavati, 2011). Moreover, (Wetzel \& Forbus, 2009) pointed out that, as per instructors, there is a level of difficulty in communicating the working of a design, made by students, through a sketch. Behaviour simulation of product concepts through an intuitive sketching interface is shown in (Onkar \& Sen, 2012). A recent study also shows that sketching supports knowledge preordering in the design process (Brun, Masson, \& Weil, 2016 ). Many of these computer based sketching support tools for conceptual design are predominantly focused on the geometric/shape information and neglects other information like gestures and annotations. The work presented in this paper highlights the importance of annotations and explores its necessity in developing computational support to conceptual design phase in product design. Annotations are important in sketching because they are the cues that convey information regarding the articulations in the product concepts.

Effort is defined as the physical or mental exertion to do a particular task/action (Cambridge, 2004). (Fleishman, Gebhardt, \& Hogan, 1986) has mentioned how effort is defined from the perspective of work physiologists, ergonomists and industrial engineers. (Fleishman, Gebhardt, \& Hogan, 1986) attempted to integrate psychophysical, physiological, and perfomance indices to estimate the effort during a physical activity. It is mentioned in (Riva, Waterworth, Waterworth, \& Mantovani, 2011) that mental effort is easily transformed into action based on 
the goal of the designer. In particular, during conceptual stage, relation between mental effort, fatigue and concentration can be found in (Nguyen, Nguyen, \& Zeng, 2018). But in the context of sketching activity, there is no relation between effort and design actions can be found in literature as per author's knowledge.

The intention of the present work is to extract intuitive visual and non-visual cues used by designers while communicating the articulations of the concept in a product sketch. For this, a design experiment has been conducted to study the sketching activity. Section 3 gives the characterization of effort of sketching activity in terms of number of design actions. Section 4 mentions the details about the methodology of the design experiment and characterization of the important parameters that must be observed in the design experiment. Section 5 contains how the data has been processed from the experiment. Section 6 gives the analysis and results of the design experiment. Discussion and conclusion are given in section 7 and 8 respectively.

\section{Characterization of Effort in Sketching Activity}

If there is a series of actions, then the total effort would be the sum of the efforts of all actions. Applying the definition in the context of a sketching. The series of design actions, in a sketching activity, is to generate a concept for a given problem statement. For the total effort involved in the sketching activity is equal to the sum of efforts involved in design actions. For simplicity, the complexity in each design action is not considered in this work. For example, efforts applied in the design actions of depiction of a circle and gear are considered equal although both require different quantities of energies and time of drawing (if they are done by hand using a pencil/pen on a paper).

If a sketching activity, say ' $i$ ', is considered which consists of number of design actions, ' $n_{i}$ '; then, the effort $\left(E_{i}\right)$ to perform the ' $i$ 'th' sketching activity is given by the following equation.

$$
E_{i}=E_{1}+E_{2}+E_{3}+\cdots+E_{n_{i}}
$$

But according to the assumption, as mentioned earlier, the complexity of a design action is not considered. This implies that energy requirements to sketch any depiction is the same. Therefore,

$$
\begin{gathered}
E_{1}=E_{2}=E_{3}=\cdots=E_{n_{i}} \\
E_{i}=n_{i} E_{1}
\end{gathered}
$$

From equation (3), it is concluded that the effort involved in the sketching activity is directly proportional to the number of design actions.

$$
E_{i} \propto n_{i} \quad(4)
$$

Hence, it is considered that the more actions in a particular sketching activity, more the effort. Now, from a design experiment conducted it is essential to extract the fraction of effort involved in communicating the functionality of the product sketch compared to the total effort put in the sketching activity. For this,

$$
\begin{aligned}
E_{p} & =n_{p} E_{p} \\
E_{t} & =n_{t} E_{t}
\end{aligned}
$$

Where,

$E_{p}=$ Effort applied by the designer to communicate the functionality of the product sketch through the design actions,

$n_{p}=$ Number of design actions required to communicate the functionality of the product sketch,

$E_{t}=$ Total effort applied by the designer to communicate the functionality of the product sketch through the design actions throughout the sketching duration. The total time taken 
by the designer wherein to generate the concept using the product sketch is called sketching duration $(t)$,

$n_{t}=$ Number of design actions required to communicate the functionality of the product sketch throughout the sketching duration.

$$
\frac{E_{p}}{E_{t}}=\frac{n_{p}}{n_{t}}
$$

Here, in equation (7), the left hand side gives the fraction of effort applied by the designer to communicate the functionality and the right hand side gives the fraction of design actions required to communicate to functionality of the product. This implies that if left hand side is estimated so is right hand side simultaneously. The next section gives the methodology on the estimation of number design actions in a sketching activity.

\section{Methodology}

A design experiment is conducted to understand sketching activity. The data obtained is analyzed in two ways as follows:

1. To enlist the various cues that help the designer to communicate the functionality of the product sketch

2. To estimate the fraction of effort made by the designer to communicate the functionality of the product sketch

\subsection{Experimental Procedure}

The steps to study the sketching activity are as follows.

1. The designers were asked to generate ideas for a product design problem which involves relative motion of its components (a stapler with a unique sequence of functionality)

2. Identify the various cues that are used by the designer to communicate the functionality of the concepts through sketching during the design experiment.

3. Estimate the number of design actions for each category viz., $n_{p}$ and $n_{t}$.

4. Estimate the fraction of design actions $\left(n_{p} / n_{t}\right)$ required to communicate the functionality of concept of the product sketches and compare between different subjects.

To understand various cues and generate the data to estimate the fraction of effort (as mentioned in equation (7)), a setup was arranged for conducting experiments and is explained in the following subsections.

\subsection{Experimental Setup}

An experiment was conducted to estimate the fraction of effort required in communicating the relative motion of the components in their sketches. Four Master students studying Product Design and Engineering specialization ( 3 males and 1 female) were selected for the experiment. The subjects were in the age group ranging from 22 to 25 years. All the subjects were given the same problem statement as "To design a stapler where the first action is used to store energy and release it to give an impact for stapling" and they were asked to generate concepts through pen/pencil- paper sketching. The subjects were given five minutes to think about the problem statement and clarify any doubts with the experimenter. After this they begin with the idea generation through sketching. The experimental setup consisted of a classroom chair with a 
drawing pad in a well-lit room. Pencils, A3 sheets and other stationery were provided to the subjects. The sketching process was audio and video recorded. A video camera was installed using a tripod in front of the chair, pointing towards paper, such that the camera would capture the sketching process. Each subject was called individually. The subjects were instructed to think aloud their thoughts while sketching. Also, they were asked to represent the working of the product with as much detail as possible through their sketches. The accuracy or correctness of the design ideas were not the criteria for evaluation.

\subsection{Characterization of Coding Scheme}

Sketching is a highly dynamic activity where physical and cognitive actions happen simultaneously. The sketching videos were analysed for actions used to communicate behaviour and functionality of the concept in their sketches. They are listed in table 1. Basically, action types 1,2 and 4 are non-verbal and type 3 is verbal. The verbal action was a consequence of think-aloud instruction that was given before beginning the experiment.

Table 1. Actions to communicate the functionality of a product sketch as observed in the experiment

\begin{tabular}{|c|c|c|c|}
\hline Type & Actions & Examples & $\begin{array}{c}\text { Action } \\
\text { Categories }\end{array}$ \\
\hline 1 & Annotations & Lines, circles, arrows, redrawing same & D-action \\
\hline 2 & Gestures & $\begin{array}{c}\text { Movement of pen/hand, move elements or } \\
\text { gesture configuration }\end{array}$ & M-action \\
\hline 3 & Utterances & Utterances while designing or explaining & Not coded \\
\hline 4 & $\begin{array}{c}\text { Written } \\
\text { sentences }\end{array}$ & Written words to explain their functionality & D-action \\
\hline
\end{tabular}

The effort involved in sketching activity cannot be directly ascertained by observing the sketching video. Hence, a reliable coding scheme needs to be adopted for a detailed analysis. One such coding scheme to analyse sketches is presented in (Suwa, Purcell, \& Gero, 1998), but, this scheme was primarily devised to study architectural conceptual sketches. Since the present work is related to product concept sketches, the coding scheme has been modified to suit the analysis. According to (Suwa, Purcell, \& Gero, 1998), design actions are divided into four categories viz. physical, perceptual, functional and conceptual. Out of these, only physical and F-actions are modified and keeping the meaning of others intact. The physical actions contain D-action, L-action and M-action. The D- action contains the sketch and written sentences that describe the function. The $\mathrm{M}$-action contains the gestures by hand and movement of the pen.

\subsubsection{Characterization of F-action}

The functional action (F-action) in (Suwa, Purcell, \& Gero, 1998) has been defined as two classes

1. Interactions between designed artefacts and people and

2. Interactions between designed artefacts and surrounding natural resources.

Since the work analysis does not involve interaction with people nor natural environments but rather interactions with designed artefacts themselves, the authors recoded the functional action in the context of product design. So "any motion interaction between depicted artefacts" is termed as functional and rest of the subcategories (see the table 4 of (Suwa, Purcell, \& Gero, 1998)) remain the same. For example, consider the scythed chariot in Figure 1(b). The swords, at the front, rotate about an axis perpendicular to the ground which is held over a box and 
connected to the wheels. Hence, if a designer explains the functionality of these swords, then the design action will be coded as F-action.

The other design actions i.e. perceptual (P-action) and conceptual (E, G and K-actions) are not modified (see (Suwa, Purcell, \& Gero, 1998) for their meanings). Verbal utterances have not been considered for the coding even though it is a physical action because it can be done simultaneously with $\mathrm{D}$ and M-action. The omission of verbal is done primarily to list out mutually exclusive design actions. For example, while the designer is sketching hand gestures and movement of the pen to describe the functionality of the product sketch cannot happen simultaneously. Whereas one can verbally communicate simultaneously while gesturing, moving the pen and drawing.

\section{Data Processing}

During the experiment the subjects were instructed to think aloud (van Someren, Barnard, \& Sandberg, 1994) their thoughts while performing actions. Every action in a design process (concept design using sketches) has a purpose. The sketching process and the utterances were audio-video recorded. Final sketches are obtained as shown in Figure 2 for all the four subjects. The utterances have been transcribed (as raw data) and processed. A modified coding scheme as described in section 3.4, was used to classify this raw data into a more meaningful data.

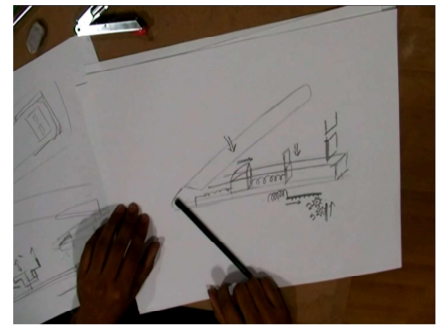

(a)

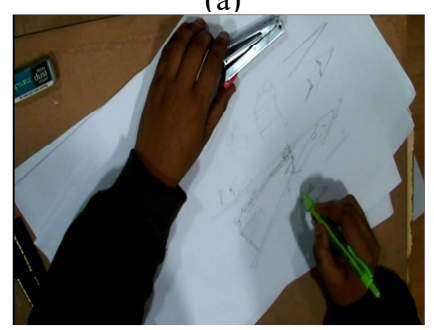

(c)

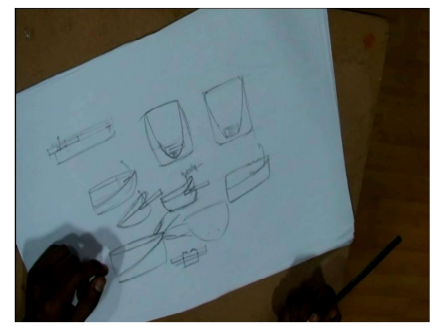

(b)

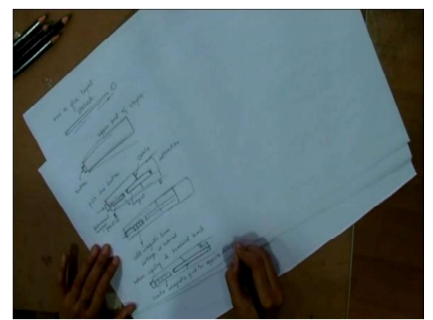

(d)

Figure 2: Final sketches of (a) Subject 1 (b) Subject 2 (c) Subject 3 (d) Subject 4.

A part of the processed data along with design actions have been shown in Table 2. Such tables are made for all the four subjects. Due to space constraints the processed data tables are not shown in the paper. Protocol for analysis has been the major technique to examine cognitive processes in design. A part of the raw data was segmented into according to their respective categories for the subjects as shown in Table 2. The text in second column shows the segment action in the video (see definition of segment from (Suwa, Purcell, \& Gero, 1998)). The third column contains the phrase uttered by the designer by which his/her intention is known to the experimenter. The text in the fourth column is the interpretation of total design action in the author's own words. Based on this, the design action has been coded as physical (D, L, or M), perceptual $(\mathrm{P})$, functional $(\mathrm{F})$ or conceptual $(\mathrm{E}, \mathrm{G}$ or $\mathrm{K})$ action in the fifth column. 
Table 2: Excerpt of coding scheme of subject 1

\begin{tabular}{|c|c|c|c|c|}
\hline $\begin{array}{l}\text { Segment } \\
\text { No. }\end{array}$ & Segment 'Si' (Action in Video) & Phrase & Interpretation/Comment & Design Action \\
\hline \multirow{3}{*}{1} & \multirow{3}{*}{ Draws two jaws on the paper } & \multirow{3}{*}{ Silence } & \multirow{3}{*}{ Only sketching } & \\
\hline & & & & 1. D \\
\hline & & & & 2. F (1) \\
\hline 2 & & $\begin{array}{l}\text {....considering this two jaws } \\
\text { and }\end{array}$ & & 3. P \\
\hline \multirow[b]{2}{*}{3} & \multirow{2}{*}{$\begin{array}{l}\text { Shows the lower jaw that } \\
\text { contains the staples }\end{array}$} & \multirow{2}{*}{$\begin{array}{l}\text { this being the one loaded with } \\
\text { staplers }\end{array}$} & \multirow{2}{*}{$\begin{array}{l}\text { He introduces a new element called } \\
\text { staples and tells that it is placed } \\
\text { inside the lower jaw. So basically, } \\
\text { he is grouping staples and lower } \\
\text { jaw }\end{array}$} & 4. M \\
\hline & & & & 5. $\mathrm{P}$ \\
\hline \multirow{2}{*}{4} & \multirow{2}{*}{$\begin{array}{l}\text { Shows free end of the stapler } \\
\text { with his finger }\end{array}$} & \multirow{2}{*}{$\begin{array}{l}\text { and this is the one which is the } \\
\text { free end }\end{array}$} & \multirow{2}{*}{$\begin{array}{l}\text { He just looks at the new depicted } \\
\text { element and declares or names it as } \\
\text { free end. }\end{array}$} & 6. M \\
\hline & & & & 7. $F(1)$ \\
\hline 5 & Draws lines to represent the canal & $\begin{array}{l}\text { this already has a canal like } \\
\text { structure }\end{array}$ & $\begin{array}{c}\text { Creates a new element in already } \\
\text { existing entity }\end{array}$ & 8. D \\
\hline \multirow{3}{*}{6} & \multirow{3}{*}{$\begin{array}{l}\text { Points to the lines to tell that } \\
\text { they are rails }\end{array}$} & \multirow{3}{*}{ so on this I am using it as rails } & Points to the canal & 9. M \\
\hline & & & $\begin{array}{c}\text { A part of the canal is perceived as a } \\
\text { rails }\end{array}$ & 10. $\mathrm{P}$ \\
\hline & & & $\begin{array}{l}\text { Rails implies that there is sliding } \\
\text { joint that is about to come }\end{array}$ & 11. F (1) \\
\hline \multirow{3}{*}{7} & \multirow{3}{*}{ Draws the slider } & \multirow{3}{*}{ On this I have a slider } & \multirow{3}{*}{ Depicts a new element } & 12. D \\
\hline & & & & 13. $P$ \\
\hline & & & & 14. F (1) \\
\hline \multirow[t]{2}{*}{8} & \multirow{2}{*}{$\begin{array}{l}\text { Points the slider and the rails and } \\
\text { gives direction of slider by } \\
\text { movement of pen }\end{array}$} & \multirow[t]{2}{*}{ this slides on these two rails } & $\begin{array}{l}\text { revisits the slider and rails and tells } \\
\text { the way they interact with each } \\
\text { other }\end{array}$ & 15. $\mathrm{P}$ \\
\hline & & & Points out to slider and rails & 16. M \\
\hline
\end{tabular}

\subsection{Inter-coder reliability}

The coding has been done twice by a single researcher within a span of four months. It has been found in the literature (Nili, Tate, Barros, \& Johnstone, 2020) that of all the reliable tests, Kirpendorff's alpha $(\alpha)$ (Krippendorff, 2004) is considered as the best. To check the reliability of the current modified coding scheme mentioned in section 4.3, a macro proposed in (Hayes \& Krippendorff, 2007) using IBM Statistics Package for the Social Sciences (SPSS) (IBM Corp, 2017) has been used. Correspondingly, subject's 1, 2, 3 and 4 estimated inter-coder reliability $\alpha$-values are $0.69,0.81,0.92$ and 0.89 . The inputs to the macro are the nominal data as shown in column 5 of Table 2 . The design actions have the characteristics of nominal data and are of multiple categories (D, L, M, P, F-actions etc.). Here, no missing codes is considered. The first and second coding of design actions are taken as two observations by the same researcher but at two different times. As per (Krippendorff, 2004), there is no standard acceptable level of reliability, but $\alpha<0.67$ is not acceptable. As all the subject's $\alpha$ values are above 0.67 , it can be concluded that the coding has been done in a reliable way without much disagreements between two observations.

\section{Data Analysis}

The sketching activity in the video is discretized into a series of design actions. An example is shown in Table 2. Such tables are generated for all the four videos of the experiment. From the Table 2 , every segment contains multiple action categories. Hence, for very segment ' $S_{i}$ ' there exist several design actions say ' $n_{i}$ '. On observation, there are certain segments that contain F- actions and others do not. Consider the segments that contain F-actions (see (Suwa, Purcell, \& Gero, 1998) for F-action meaning) and let the number of physical actions in each of them be ' $k_{i}$ '. The main objective here is to estimate the percentage of number of physical actions required to describe the functionality of the concept in the sketch $\left(n_{p}\right)$ from each table for each subject (see section 3 for characterization). The following are the steps followed for the estimation. 
1. Consider the last column of the table (as in Table 2). The design actions are sequentially numbered starting from first design action of the first segment to the last action of the last segment. The last number gives the total number of design actions $\left(n_{t}\right)$.

$k_{i}=$ Number of physical actions in the segment where $\mathrm{F}-$ action is coded

2. Sum all the numbers in the brackets of F-action which gives number of physical actions that describe the functionality of concept of the sketch $\left(n_{p}\right)$.

$$
n_{p}=\sum_{i=i}^{n_{t}} n_{i}
$$

Where,

$$
n_{i}=\left\{\begin{array}{c}
=0, \text { if } S_{i} \text { does not contain } F-\text { action } \\
k_{i}+1
\end{array}\right.
$$

3. Calculate $\left(n_{p} / n_{t}\right)$ to obtain the percentage of physical actions in communicating the functionality of concept in the sketch.

The fundamental idea of the above procedure is to calculate the total number of physical actions that describe the functionality of the artefacts or the sketch, which, is equal to sum of all the physical actions that describe the functionality of the sketch or artefacts in each segment. The physical actions that describe the functionality in each segment is the value inside the bracket of F-action plus 1. For example, the total number of functional actions described by physical actions in table 2 is $(1+1)+(1+1)+(1+1)+(1+1)=8$. These values have come from the design actions $(1,2),(6,7),(9,11)$ and $(12,14)$ respectively. For this excerpt of coded data, in table 2 , the percentage/fraction of effort required to describe the functionality of the sketch is $8 / 16=0.5$ or $50 \%$.

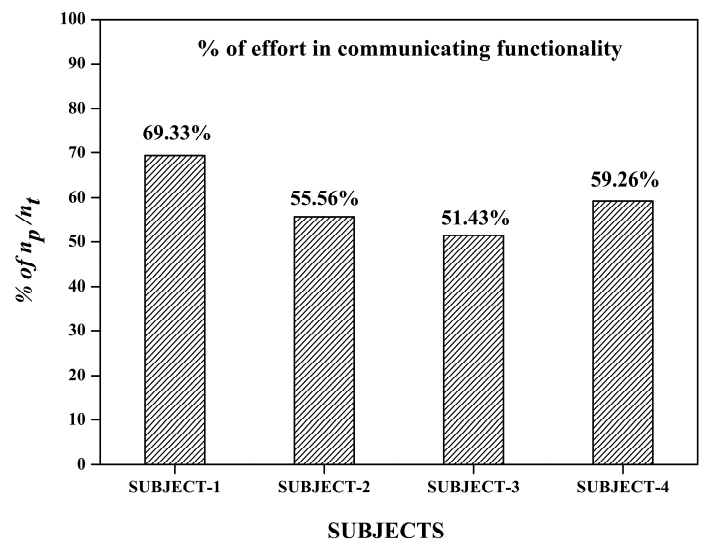

(a)

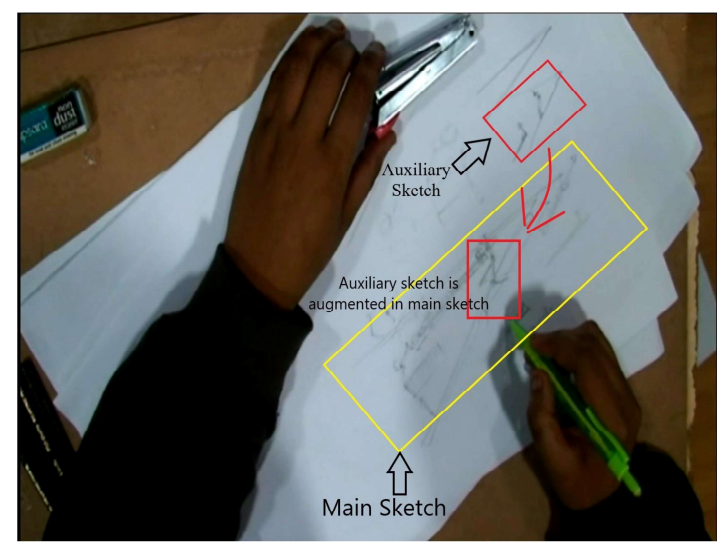

(b)

Figure 3: (a) Comparison of percentages of physical actions that describe functionality of sketch

(b) Main and Auxiliary sketch of Subject 3

Similarly, in this way, for all the subjects the percentages of physical actions to explain the functionality of the sketch are obtained as shown in Fig. 3(a). Two important things are to be noted before concluding the results. These are as follows. 
Table 3: Experiment Details

\begin{tabular}{|c|c|c|c|c|c|c|}
\hline Subject & $\boldsymbol{t}$ (seconds) & $\boldsymbol{n}_{\boldsymbol{p}}$ & $\boldsymbol{n}_{\boldsymbol{t}}$ & $\boldsymbol{n}_{\boldsymbol{p}} / \boldsymbol{n}_{\boldsymbol{t}}$ & $\mathbf{A}_{\mathrm{U}}$ & $\mathbf{1} / \mathbf{A}_{\mathbf{U}}$ \\
\hline 1 & 213 & 52 & 75 & $69.33 \%$ & 0.35 & 2.87 \\
\hline 2 & 319 & 35 & 63 & $55.55 \%$ & 0.2 & 5.06 \\
\hline 3 & 363 & 36 & 70 & $51.4 \%$ & 0.19 & 5.18 \\
\hline 4 & 439 & 16 & 27 & $59.26 \%$ & 0.06 & 16.25 \\
\hline
\end{tabular}

1. Physical actions are a category of design actions involved in sketching activity (see section 4.3) and

2. The relation between fractions of design actions involved in communicating the functionality of the concept in the product sketch in same as that of the fraction of effort (derived in equation 7).

So, from above two points it can be seen from Fig. 3(a) that more than 50\% of the effort is spent in describing the functionality of the concept of sketch. Table 3 gives details of the experiment.

The sixth column Au (in Table 3) indicates the number of physical actions per unit time. Of these, subject 1 has the highest which is 0.35 physical actions per second. This measure can be seen in the sketching video explicitly. The subject has a high physical activity while sketching i.e. rapid change in mode of gesturing, drawing, moving the pen etc. Similarly, the subject 4 has the lowest which is 0.06 physical actions per second. This can also be seen in the recorded video where the hand movements for drawing, gesturing etc. are comparatively low with other subjects. Here in the design experiment, the complexity or aesthetics of the drawings is not rated but rather the number of physical actions has been counted and compared relatively. The seventh column $\mathbf{1 / A U}$ (which is self-explanatory) inverse of number of physical actions per unit time i.e. time taken per physical action.

\section{Discussion}

From analyzing the sequence of actions performed by designers, it is observed that designers use annotations (words, arrows, lines, circles etc.) for making depictions on the sketches to communicate the functionality. Designer may sketch more than one view of the same artefact to explain the working of the same component. In addition, in traditional product sketching i.e. on paper, the designers tend to have a main sketch and auxiliary sketches for exploration. The main sketches are augmented whenever they gain clarity of a physical principle in auxiliary sketches. So, they tend to move between these main and auxiliary sketches which increase the physical number of actions. For example, the subject 3 in the experiment multiple auxiliary sketches and a main sketch as shown in Figure 3(b).

Evidences can be spotted in the recorded sketching video in which the designers were unable to describe the motion of the component in the sketch clearly due to its complexity. This was seen for the subject 3 . This clearly indicates the need for a facility for motion visualization of the path traced by the point, which is not possible in a traditional sketching medium.

The designers use physical actions like moving a pen, gestures, verbal sentences etc. to visualize the interactions between the elements. These conclusions are arrived at by studying 
the data generated through think-aloud protocol. Thus, it can be said that this percentage of physical actions to communicate the functionality of concept in a design process can be one of the potential quantities in assessing the quality of the design process. Even though as pointed out in section 1 about the difficulty in conveying the working of product sketches, the authors have not come across any published work about quantifying it. All the work prior to (Wetzel \& Forbus, 2009) have been based on qualitative mechanics (Forbus, Nielsen, \& Faltings, 1991). The present work aims to quantify the ratio of effort invested by the designer to convey or communicate how his/her concept works to the total effort invested in sketching the concept. The authors speculate that, if amount of the effort spent to communicate is less, then the designer will be less fatigued. Hence, it increases his/her focus on the creative aspect of generating concepts. Such a relation between effort, fatigue and concentration has been mentioned in (Nguyen, Nguyen, \& Zeng, 2018). So, there is a dire need to find new ways to communicate to justify the relative motion between the sub-components (interpreted in form of depictions on paper) of the product that is to be designed.

\section{Conclusion and Future Work}

It is concluded that the designers spend considerable effort in explaining the functionality of articulations in the concepts represented in a sketch. Of course, the measurement is based on the modified (Suwa, Purcell, \& Gero, 1998) coding scheme and on the assumptions that energies spent on the depictions are equal. Further, this effort spent is also not productive because it is found that the interpreted behavior leads to inconsistencies. For example, if the staplers of all the subjects were to physically prototyped as they are sketched, as shown in Figure 2, they will not lead to intended motion. Thus, a computer-based sketching interface which simulates the annotations would be useful so that the designers can spend more time in exploring concepts which are more practical and feasible to realize. The main aim should be to reduce the percentage of contribution of physical actions in annotations and enhance the designer's contribution to the creative aspect of the design process and not bother about the complex movements of the product.

The procedure developed in this paper will be used to compare traditional sketching and sketch- based interface being developed. It is also planned to develop guidelines for sketchbased interfaces to incorporate annotations. Once the support for motion explorations is available in sketch-based interfaces, it would be possible to compare the novelty and quality of the concepts developed through such interfaces.

\section{References}

Bilda, Z., Gero, J. S., \& Purcell, T. (2006). To sketch or not to sketch? That is the question. Design Studies, 27(5), 587-613.

Boothroyd, G., Dewhurst, P., \& Knight, W. A. (2010). Product Design for Manufacture and Assembly. CRC Press.

Brun, J., Masson, P. L., \& Weil, B. (2016 ). Designing with sketches: the generative effects of knowledge preordering. Design Science, 2(e13), 26. doi:https://doi.org/10.1017/dsj.2016.13

Cambridge. (2004). Oxford Dictionary of National Biography.

Ferguson, E. S. (1994). Engineering and the Mind's Eye. MIT Press. Sótt frá https://mitpress.mit.edu/books/engineering-and-minds-eye

Fish, J., \& Scrivener, S. (1990). Amplifying the Mind's Eye: Sketching and Visual Cognition. Leonardo, 23(1), 117-126. doi:10.2307/1578475 
Fleishman, A. E., Gebhardt, L. D., \& Hogan, J. C. (1986). The Perception of Exertion in Physical Work. Wenner-Gren Center International Symposium Series. London: Palgrave Macmillan, London. doi:https://doi.org/10.1007/978-1-349-08946-8_19

Forbus, K. D., Nielsen, P., \& Faltings, B. (1991). Qualitative spatial reasoning: The CLOCK project. Artificial Intelligence, 417-471. Sótt frá https://doi.org/10.1016/00043702(91)90116-2

Gero, J. S., \& Kannengiesser, U. (2004). The situated function-behaviour-structure framework. Design Studies, 25(4), 373-391.

Gero, J. S., \& Purcell, A. T. (1998). Drawings and the design process: A review of protocol studies in design and other disciplines and related research in cognitive psychology. Design Studies, 389-430.

Goldschmidt, G. (1991). The dialectics of sketching. Creativity Research Journal, 4(2), $123-$ 143.

Hayes, A. F., \& Krippendorff, K. (2007). Answering the Call for a Standard Reliability Measure for Coding Data. Communication Methods and Measures, 1(1), 77-89.

IBM Corp. (2017). Released 2017. IBM SPSS Statistics for Windows, Version 25.0. Armonk, NY: IBM Corp.

Jorge, J., \& Samavati, F. (2011). Sketch-based Interfaces and Modeling. London: SpringerVerlag London. doi:10.1007/978-1-84882-812-4

Krippendorff, K. (2004). Content Analysis: An Introduction to Its Methodology. SAGE.

Nguyen, P., Nguyen, T. A., \& Zeng, Y. (2018). Empirical approaches to quantifying effort, fatigue and concentration in the conceptual design process. Research in Engineering Design, 29, 393-409.

Nili, A., Tate, M., Barros, A., \& Johnstone, D. (2020). An approach for selecting and using a method of inter-coder reliability in information management research. International Journal of Information Management, 102154. doi:https://doi.org/10.1016/j.ijinfomgt.2020.102154

Norton, R. L. (2004). Design of Machinery: An Introduction to the Synthesis and Analysis of Mechanisms and Machines. McGraw-Hill Higher Education.

Onkar, P. S., \& Sen, D. (2010). Functional Segmentation of Strokes for Product Sketch Understanding. International Journal of Shape Modeling, 16, 9-38.

Onkar, P. S., \& Sen, D. (2012). Behaviour Simulation in Computer Aided Product Concept Sketching. CIRP Design 2012 (bls. 181-191). London: Springer, London. doi:https://doi.org/10.1007/978-1-4471-4507-3_18

Riva, G., Waterworth, J. A., Waterworth, E. L., \& Mantovani, F. (2011). From intention to action: The role of presence. New Ideas in Psychology, 29(1), 24-37.

Schon, D. A. (2017). The Reflective Practitioner - How Professionals Think in Action (reprint útg.). Routledge.

Suwa, M., Purcell, T., \& Gero, J. (1998). Macroscopic analysis of design processes based on a scheme for coding designers' cognitive actions. Design Studies, 19(4), 455-483.

van Someren, M. W., Barnard, Y. F., \& Sandberg, J. C. (1994). The think aloud method: a practical approach to modelling cognitive processes. London: London: Academic Press.

Wetzel, J., \& Forbus, K. (2009). Automated Critique of Sketched Mechanisms. Proceedings of the 21st Innovative Applications of Artificial Intelligence Conference, AAAI-09. 